\title{
Who Should Pay for Forwarding Packets?
}

\author{
Heiner Ackermann, Patrick Briest, Alexander Fanghänel, and \\ Berthold Vöcking
}

Abstract. We present a game-theoretic study of hybrid communication networks in which mobile devices can connect to a base station, The maximal number of allowed hops might be bounded in order to guarantee small latency. We introduce hybrid connectivity games to study the impact of selfishness on this kind of infrastructure.

Mobile devices are represented by selfish players, each of which aims at establishing an uplink path to the base station minimizing its individual cost. Our model assumes that intermediate nodes on an uplink path are reimbursed for transmitting the packets of other devices. Depending on the model, the reimbursements can be paid either by a benevolent network operator or by the senders of the packets using micropayments via a clearing agency that possibly collects a small percentage as commission.

Our main findings are these: If there is no constraint on the number of allowed hops on the path to the base station, then the existence of equilibria is guaranteed regardless of whether the network operator or the senders pay for forwarding packets. If there is an upper bound on the number of allowed hops on the uplink path, then the existence of equilibria depends on who pays for forwarding packets. If the network operator pays, then the existence of equilibria is guaranteed only if at most one intermediate node is allowed. If the senders pay for forwarding their packets, then equilibria are guaranteed to exist given any upper bound on the number of allowed hops.

Our equilibrium analysis gives a first game-theoretic motivation for the implementation of micropayment schemes in which senders pay for forwarding their packets. We further support this evidence by giving an upper bound on the "price of anarchy" for this kind of hybrid connectivity game that is independent of the number of nodes but depends only on the number of hops and the power gradient.

(C) A K Peters, Ltd.

|542-795|/08 $\$ 0.50$ per page 


\section{Introduction}

Hybrid communication networks are a promising direction in which to combine the features of wireless ad hoc networks with the advantages of wired networks guaranteeing flexible connectivity at low cost in combination with a high throughput. In such an infrastructure, mobile devices connect in an ad hoc fashion to a base station, i.e., an access point to the wired part of the network, possibly via a few hops using other mobile devices as relay stations [Frodigh et al. 01, Singh et al. 98, Tang et al. 05].

Since energy requirements increase superlinearly in the distance between two devices, the use of intermediate nodes can significantly reduce the energy consumption in comparison to directly connecting to the base station. This is of particular importance for uplink connections from the mobile devices to the base stations, since mobile devices have rather limited energy resources. Using mobile devices as relay stations, on the one hand, might also increase the quality of service (QoS) due to a reduction of interference. On the other hand, the QoS suffers from an increase in latency if packages need to be forwarded several times until they reach the wired part of the network. For this reason, only a relatively small number of hops seems to be acceptable. We focus on energy consumption while neglecting interference issues. The aspect of keeping the latency at a reasonable level is modeled by introducing hop constraints, which ensure that uplink paths are not too long.

Although the benefits of using multihop connections are convincing from a global point of view, one might ask why participants in a commercially operated network should forward packets of other participants, since this drains the battery of the forwarding node while offering no benefit to the participant, thus bringing a negative utility to that participant. The usual response is that the forwarding nodes should receive a payment for forwarding packets. Let us simplify and assume that there is perfect information about the cost of forwarding packets. More specifically, we assume that the energy consumption for sending packets between any pair of nodes is publicly known and there is a common valuation per unit energy among the players, so that intermediate nodes can be reimbursed for forwarding packets. Nodes are reimbursed for forwarding packets either by the network operator or by the senders of the packet. We assume that these payments exactly compensate the cost of packet forwarding.

We introduce hybrid connectivity games as a game-theoretic model for hybrid communication networks and study the existence, structure, and complexity of Nash equilibria in these games depending on different kinds of payment schemes. Mobile devices are represented by players that aim at connecting to an access point via an uplink path. 
We study the effects of payments on the equilibrium topologies in hybrid connectivity games depending on who is paying for reimbursing the intermediate nodes on the uplink path for forwarding packets: The network operator might pay for all reimbursements, or the senders of the forwarded packets might pay. One can imagine that the first variant can be implemented internally within the accounting system of the network operator/provider. The second variant suggests itself for an implementation using micropayments as elucidated, e.g., in [Jakobsson et al. 03]. A clearing agency realizing the micropayments possibly collects a small additional percentage as commission from the senders. We will incorporate these different variants into our model in the form of a parameter $\alpha$ describing the fraction of payments made by the senders. Here $\alpha=0$ means that the network operator completely pays for the reimbursements, whereas $\alpha \geq 1$ means that the senders reimburse the nodes forwarding their packets and the additional payment, i.e., a fraction of $\alpha-1$, goes to the clearing agency.

\section{I.I. The Model}

A hybrid connectivity game consists of a complete, edge-weighted graph $G=$ $(V, E)$ with $V=P \dot{\cup} A$ and two parameters $\alpha \geq 0$ and $h \in \mathbb{N}$. We denote by $P$ the set of players, and by $A$ the set of access points. In the following, let $n=|P|$. The edge weights are assumed to be positive, and weight $w(i, j)$ of edge $\{i, j\}$ describes the cost of transmitting a unit of data from $i$ to $j$ or vice versa.

Each player aims to establish an uplink path to an access point. To this end, player $i$ chooses a gateway $g_{i} \in V$. The idea is that $i$ sends its own packets and all other packets that it receives to $g_{i}$. Then this node forwards these packets to its gateway and so on until the packets reach an access point. The path followed by the packets to the access point is called $i$ 's uplink path.

In the following, we call a vector $s=\left(g_{1}, \ldots, g_{n}\right) \in V^{n}$ a state of the game and assume that player $i$ chooses $g_{i}$ as gateway. The cost $c_{i}(s)$ of player $i$ in state $s$ is defined as follows. If $i$ is connected via the uplink path $i=p_{0}, p_{1}, \ldots, p_{l-1}, p_{l}=a$ to the access point $a$ and this route has at most $h$ hops, i.e., $l \leq h$, then the cost of $i$ is

$$
c_{i}(s)=w\left(p_{0}, p_{1}\right)+\alpha \cdot \sum_{j=1}^{l-1} w\left(p_{j}, p_{j+1}\right) .
$$

Otherwise, the cost of $i$ is assumed to be infinitely large. We assume that each player selfishly aims at minimizing its cost. A state $s$ is called a Nash equilibrium if no player has an incentive to change its gateway.

In the following, we assume without loss of generality that there exists a single access point only, i.e., $|A|=1$. In the case of multiple access points one can 
always merge them into a single one and choose for every player the weight of the edge between the player and the new access point as the minimum over all weights between the player and each access point from the set $A$.

\subsection{Our Contribution}

First, we consider the case that the network operator pays for reimbursing the intermediate nodes, i.e., we assume $\alpha=0$. In this case, nodes can forward packets along intermediate nodes for free, so that they aim solely at minimizing the energy requirement for the first link on their path.

- We observe that if there is no hop constraint (i.e., $h=n$ ), then hybrid connectivity games with $\alpha=0$ always have pure equilibria. A similar observation has been made in [Eidenbenz et al. 03]; see Section 1.3.

- The situation changes, however, when hop constraints are introduced. We prove that the existence of equilibria is guaranteed only for $h \in\{1,2\}$. For any $h \geq 3$, there is an instance of the hybrid connectivity game with $\alpha=0$ that does not have an equilibrium.

Let us remark that both existence proofs are constructive and yield algorithms computing equilibria efficiently.

Second, we study the case that intermediate nodes are reimbursed for forwarding packets by the senders of the packets via an agency that might collect a small percentage as commission, that is, we assume $\alpha \geq 1$. In this case, our analysis shows that these games always have pure equilibria. Again our proof is constructive and yields an efficient algorithm for computing an equilibrium.

We view our result as the first game-theoretic evidence that senders rather than a benevolent network operator should pay for forwarding their packets. This is true even if this causes some overhead for implementing the accounting and payment, since our positive results about the existence of equilibria hold even if there is a clearing agency taking some percentage as commission.

We complete our analysis by studying the price of anarchy (PoA) for hybrid connectivity games. We start by presenting examples for different variants of hybrid connectivity games, showing that the PoA is unbounded for general cost matrices. For this reason, we restrict ourselves to cost matrices generated by power graphs with an underlying Euclidean embedding. Assuming that senders pay for forwarding their packets, as suggested by our preceding equilibrium analysis, we obtain an upper bound of $h^{\beta-1}$ for the PoA, with $h$ denoting the hop constraint and $\beta$ the power gradient. Hence, the PoA is independent of the size of the network. 


\section{I.3. Related Work}

In recent years, various papers have studied wireless networks from a gametheoretic perspective. Among others, [Altman and Altman 03], [Heikkinen 06], and [Krishnaswamy 02] observe that due to the increased complexity of modern wireless networks, resource-management tasks should be shifted from the wired part of the infrastructure to the mobile devices. Such management tasks include power assignments to, and channel allocation of, mobile devices. These researchers propose to study such problems in the framework of potential games, and they present potential functions showing that best-response dynamics converge to stable assignments. Extended discussion of these and other models can be found in [Srivastava et al. 05].

Most closely related to our work is [Eidenbenz et al. 03], which introduces several topology control games. In such games, selfish mobile devices aim to be connected to specified sets of other devices at the lowest cost. Although they suggest that senders should pay for forwarding their packets, they do not take into account the effects of these payments on the preferences of the players. In our notation, they assume $\alpha=0$. They show that the connectivity games they consider do not possess Nash equilibria in general. However, if each device wishes to be connected to every other device, then the existence of equilibria is guaranteed. Hop constraints are not taken into account.

Different approaches encouraging mobile devices to forward packets are presented, for instance, in [Anderegg and Eidenbenz 03, Eidenbenz et al. 07, Jakobsson et al. 03, Zhong et al. 05]. In [Jakobsson et al. 03] a micropayment scheme is presented, whereas other works discuss incentive-compatible payment schemes [Anderegg and Eidenbenz 03, Eidenbenz et al. 07, Zhong et al. 05].

Other branches of research related to our work are network design and network formation games [Albers et al. 06, Anshelevich et al. 03, Bala and Goyal 00, Demaine et al. 07, Fabrikant et al. 03]. In [Anshelevich et al. 03], a networkcreation game is considered in which players represent subsets of nodes of a graph and can contribute toward the purchase of fixed-price edges. Among other results they show that if each player wants to connect a single node to some common source, there exist socially optimal Nash equilibria, or more formally, the price of stability in this game is 1 .

Another basic model of network formation, in which each node is represented by a selfish agent, is introduced in [Fabrikant et al. 03]. Every player can create incident links to other nodes and incurs a cost that equals $\alpha$ times the number of created links plus the sum of distances to all other nodes. [Fabrikant et al. 03] presented first results on the price of anarchy in these games. Several extensions and improvements on these results appear in [Corbo and Parkes 05, Albers et al. 06, Demaine et al. 07]. 


\section{Payments by the Network Operator}

In this section, we consider hybrid connectivity games with $\alpha=0$, that is, we consider games in which the network operator reimburses players for forwarding packets. First we consider games without hop constraints.

Theorem 2.I. Every hybrid connectivity game with $\alpha=0$ and without hop constraints possesses a Nash equilibrium that can be computed efficiently.

Proof. Consider an improvement sequence whereby players sequentially choose new gateways decreasing their costs. Since a player's improvement does not change any other players' costs, each player chooses each gateway at most once. Therefore, starting from any initial state, after at most $(n-1)^{2}$ improvement steps an equilibrium is reached.

We now turn our attention to hybrid connectivity games with $\alpha=0$ and hop constraints. Recall that in this case an uplink path is feasible only if the number of hops on this path does not exceed the hop constraint $h$. First, note that we cannot apply the arguments of the previous proof to games with hop constraints. This is because an improvement of a single player may violate the hop constraints of other players, and thus the costs of other players may increase to infinity.

In the following, we prove that every game with hop constraint $h \in\{1,2\}$ possesses a Nash equilibrium. In case of $h=1$, all players connect to the access point, an arrangement that is obviously a Nash equilibrium. In case of $h=2$, we present an efficient algorithm for computing an equilibrium. However, as shown in Observation 4.1, other equilibria besides this one may exist. Additionally, we observe that for every fixed hop constraint $h \geq 3$ there exists a game that does not possess a Nash equilibrium.

Theorem 2.2. Every hybrid connectivity game with $\alpha=0$ and hop constraint $h=2$ possesses a Nash equilibrium, which can be computed efficiently.

Proof. In the following, we present an efficient algorithm for computing a Nash equilibrium of such a game. The algorithm proceeds in two phases. Without loss of generality, assume that $w(1, a) \leq w(2, a) \leq \cdots \leq w(n, a)$.

Phase 1: Initially, all players are unconnected. The algorithm then processes the players $1, \ldots, n$ in that order and connects player $i$ to the access point if there exists no player $j<i$ with the following properties: Player $j$ has already been connected to the access point, and $w(i, j)<w(i, a)$. If such a player $j$ exists, player $i$ remains unconnected. 
Phase 2: All players who have not been connected in the first phase are connected to their closest gateway.

Players who were connected in the second phase do not have an incentive to change their gateways, since they cannot decrease their cost by choosing players connected in the second phase as their gateways, because of the hop constraint. Thus, it remains to show that none of the players who were connected in the first phase have an incentive to change their gateways. To obtain a contradiction, assume that player $i$ has an incentive to choose a new gateway $g_{i}$. Note that $g_{i}$ was connected in the first phase, too. Thus $g_{i}>i$, since otherwise, $i$ would not have been connected in the first phase. We conclude that

$$
\begin{array}{rlrl}
w(i, a) & \leq w\left(g_{i}, a\right), & & \text { since } i<g_{i}, \\
w\left(g_{i}, a\right) \leq w\left(i, g_{i}\right), & & \text { since } g_{i} \text { connected to the access point, } \\
w\left(i, g_{i}\right)<w(i, a) & & \text { since } i \text { has an incentive to connect to } g_{i} .
\end{array}
$$

Finally, we obtain a contradiction, since the inequalities implies that $w\left(i, g_{i}\right)<$ $w\left(i, g_{i}\right)$.

Next, we show that in general, the existence of Nash equilibria cannot be extended to games with hop constraint $h \geq 3$.

Theorem 2.3. For every integer $h \geq 3$, there exists a hybrid connectivity game with $\alpha=0$ and hop constraint $h$ that does not possess a Nash equilibrium.

The very technical construction of these games can be found in the appendix, Section 6.

\section{Payments by the Senders}

In this section, we consider hybrid connectivity games with $\alpha \geq 1$, and prove that every such game possesses a Nash equilibrium even in the presence of a hop constraint. Our proof applies arguments similar to those used in showing the correctness of Dijkstra's shortest path algorithm.

Theorem 3.I. Every hybrid connectivity game with $\alpha \geq 1$ and hop constraint $h \geq 1$ possesses a Nash equilibrium that can be computed efficiently.

Proof. First, we present an efficient iterative algorithm for computing an equilibrium. The algorithm works as follows. Initially, none of the players is connected. 
The algorithm then selects player $i$ such that $i=\operatorname{argmin}_{j \in P}\{w(j, a)\}$ and connects it to the access point $a$. Without loss of generality, assume that $i=1$. This state corresponds to an equilibrium when all unconnected players are ignored. The algorithm then proceeds as follows. Suppose the algorithm has already connected the players $1, \ldots, i-1$ via uplink paths to the access point such that none of them has an incentive to change its gateway. For every unconnected player $j$, let $C_{j}(i-1)$ be the cost of a cheapest uplink path via players $1, \ldots, i-1$ that does not violate the hop constraint. Let $i$ be the player such that $C_{i}(i-1)$ is minimal, i.e., $i=\operatorname{argmin}_{j \in P \backslash\{1, \ldots, i-1\}} C_{j}(i-1)$. The algorithm then connects $i$ via its cheapest path. In order to prove that we obtain a Nash equilibrium with $i$ players, we claim that the cost $C_{i}(i-1)$ is not less than the cost of the uplink path of each of the players $1, \ldots, i-1$, that is, $C_{i}(i-1) \geq C_{j}(i-1)$ for $1 \leq j \leq i-1$.

We prove our claim by induction on the number of players. First, note that the algorithm connects player 1 first, i.e., one of the players with the cheapest edge to the access point. By the construction of our algorithm, $C_{2}(1) \geq w(1, a)$. As our induction hypothesis let the first $i-1$ players be connected by the algorithm and let $C_{k}(k-1) \geq C_{j}(k-1)$ for all $j, k, 1 \leq j<k \leq i-1$. We fix a player $j<i$. Since the algorithm connected player $j$ before player $i$, we have $C_{i}(j-1) \geq$ $C_{j}(j-1)$. Now we distinguish the following two cases. In the first case, player $i$ chooses $j$ as its gateway. In the second case, it chooses a device $k \neq j$ or the access point itself as its gateway. In the first case, $C_{i}(i-1) \geq C_{j}(i-1)$, since $C_{i}(i-1)=w(i, j)+(\alpha-1) \omega_{j}+C_{j}(i-1) \geq C_{j}(i-1)$, where $\omega_{j}$ denotes the cost of the first edge on the uplink path of player $j$. The second case divides into two subcases. If $k<j$, then $C_{i}(i-1)=C_{i}(j-1) \geq C_{j}(j-1)=C_{j}(i-1)$. Otherwise, if $k>j$, then $C_{k}(k-1) \geq C_{j}(k-1)$ by the induction hypothesis. Then $C_{i}(i-1)=w(i, k)+(\alpha-1) \omega_{k}+C_{k}(k-1) \geq C_{k}(k-1) \geq C_{j}(k-1)=C_{j}(i-1)$.

We conclude that no player $j<i$ has an incentive to choose player $i$ as gateway.

In the following, we assume that each player has a strict preference in the case of a tie of the cost of two paths. We then show that the Nash equilibrium is unique, except for sets of players with mutual distance zero. Again, our proof applies arguments similar to those used for showing the correctness of Dijkstra's shortest path algorithm.

Theorem 3.2. Consider a hybrid connectivity game with $\alpha \geq 1, w_{i, j}>0$, for all $i, j \in$ $V$, hop constraint $h \geq 1$, and suppose that every player has strict preferences in the case of a tie for the cost of two paths. Then the Nash equilibrium of the game is unique. 
Proof. In the following, we denote the gateway of player $i$ in state $s$ by $g_{i}(s)$. Suppose that there exist two Nash equilibria $s_{1}$ and $s_{2}$. Then there exists at least one player $i$ such that $g_{i}\left(s_{1}\right) \neq g_{i}\left(s_{2}\right)$. Among all such players, let player $k$ be the one with smallest index with respect to the following order of the players. Without loss of generality, assume that

$$
c_{1}\left(s_{1}\right) \leq c_{2}\left(s_{1}\right) \leq \cdots \leq c_{n}\left(s_{1}\right)
$$

Thus, the players $1, \ldots, k-1$ are connected in the same manner in both equilibria, i.e., for every $j<k$, we have $g_{j}\left(s_{1}\right)=g_{j}\left(s_{2}\right)$. Next, we distinguish two cases, both of which lead to a contradiction.

Case 1: $g_{k}\left(s_{2}\right) \in\{a, 1, \ldots, k-1\}$. In this case, player $k$ has an incentive to change its gateway either in $s_{1}$ or in $s_{2}$. In both cases it switches to the gateway it has in the other state, which is a feasible choice, since the players $1, \ldots, k-1$ are connected in the same manner in both equilibria. If $k$ has the same cost in both states, then we obtain a contradiction to our assumption that $k$ has a strict preference in the case of a tie. However, if the cost in one of the two states is strictly less than in the other one, then $k$ can strictly decrease its costs in the more expensive one.

Case 2: $g_{k}\left(s_{2}\right) \in\{k+1, \ldots, n\}$. In this case, we distinguish three subcases.

(a) $c_{k}\left(s_{1}\right)<c_{k}\left(s_{2}\right)$ : Since the players $1, \ldots, k-1$ are connected in the same manner in both equilibria, player $k$ has an incentive to switch to $g_{k}\left(s_{1}\right)$ in $s_{2}$. This strictly decreases its cost.

(b) $c_{k}\left(s_{1}\right)>c_{k}\left(s_{2}\right)$ : Let $k^{\prime}$ be the last player on the uplink path from $k$ to the access point in $s_{2}$ such that $k^{\prime} \in\{k+1, \ldots, n\}$ and $g_{k^{\prime}}\left(s_{2}\right) \in\{a, 1, \ldots, k-1\}$. Note that such a player always exists. In order to find such a $k^{\prime}$, observe that either $g_{k}\left(s_{2}\right)$ has the desired properties, or $g_{g_{k}\left(s_{2}\right)}\left(s_{2}\right) \notin\{a, 1, \ldots, k-1\}$. If $g_{g_{k}\left(s_{2}\right)}\left(s_{2}\right) \in\{a, 1, \ldots, k-1\}$, then all subsequent players on the path belong to this set as well. Thus, $k^{\prime}=g_{k}\left(s_{2}\right)$. If $g_{g_{k}\left(s_{2}\right)}\left(s_{2}\right) \notin\{a, 1, \ldots, k-1\}$, then $g_{g_{k}\left(s_{2}\right)}\left(s_{2}\right)$ is the next candidate to be considered. Repeating this finally yields $k^{\prime}$ with the desired properties, since the path terminates at the access point $a$.

Now we observe that $c_{k^{\prime}}\left(s_{2}\right)<c_{k}\left(s_{2}\right)$, since $w_{i, j}>0$ for every $i, j$, and since $\alpha \geq 1$. Additionally, we observe that $c_{k}\left(s_{1}\right) \leq c_{k^{\prime}}\left(s_{1}\right)$, due to (3.1) and since $g_{k}\left(s_{2}\right) \in\{k+1, \ldots, n\}$. Thus,

$$
c_{k^{\prime}}\left(s_{2}\right)<c_{k}\left(s_{2}\right)<c_{k}\left(s_{1}\right) \leq c_{k^{\prime}}\left(s_{1}\right)
$$

In this case, however, player $k^{\prime}$ has an incentive to change its gateway to $g_{k^{\prime}}\left(s_{2}\right)$ in $s_{1}$, since the players $1, \ldots, k-1$ are connected in the same manner in $s_{1}$ and $s_{2}$. 
(c) $c_{k}\left(s_{1}\right)=c_{k}\left(s_{2}\right)$ : Since $w_{i, j}>0$ for every $i, j$, and since $\alpha \geq 1$, we conclude that $c_{g_{k}\left(s_{2}\right)}<c_{k}\left(s_{2}\right)$. Furthermore, we conclude from (3.1) and the assumption that $g_{k}\left(s_{2}\right) \in\{k+1, \ldots, n\}$ that $c_{k}\left(s_{1}\right) \leq c_{g_{k}}\left(s_{1}\right)$. Thus

$$
c_{g_{k}\left(s_{2}\right)}\left(s_{2}\right)<c_{k}\left(s_{2}\right)=c_{k}\left(s_{1}\right) \leq c_{g_{k}}\left(s_{1}\right) .
$$

Now consider the gateway $g_{g_{k}\left(s_{2}\right)}\left(s_{2}\right)$ of player $k$ in $s_{2}$. Suppose now that $g_{g_{k}\left(s_{2}\right)}\left(s_{2}\right) \in\{a, 1, \ldots, k-1\}$. In this case, we can apply case 1 to $g_{g_{k}\left(s_{2}\right)}\left(s_{2}\right)$, which implies that one of the two states $s_{1}$ and $s_{2}$ is not an equilibrium. If, however, $g_{g_{k}\left(s_{2}\right)}\left(s_{2}\right) \in\{k+1, \ldots, n\}$, then we can apply the second subcase of case 2 to $g_{g_{k}\left(s_{2}\right)}\left(s_{2}\right)$. This is possible because $(3.2)$ implies that $c_{g_{k}\left(s_{2}\right)}\left(s_{1}\right)>c_{g_{k}\left(s_{2}\right)}\left(s_{2}\right)$. We obtain that $g_{g_{k}\left(s_{2}\right)}\left(s_{2}\right)$ has an incentive to change its gateway in $s_{1}$. It follows that the equilibrium is unique.

From a theoretical point of view, it would also be interesting to consider games with $\alpha \in] 0,1[$. In this case reimbursements would be shared among the sender and the network operator. However, we conjecture that for every hop constraint $h \geq 3$ and $\alpha \in] 0,1[$, there exists a hybrid connectivity game that does not have an equilibrium.

\section{Price of Anarchy}

In this section we provide some results on the price of anarchy (PoA) in hybrid connectivity games. As usual, the PoA is the ratio between the sum of the players' costs in a worst Nash equilibrium and in a socially optimal state. First, we present examples for $\alpha \in\{0,1\}$ showing that in general, the PoA cannot be bounded by any constant. In the case of games with $\alpha=0$ this is true for any $h \geq 2$ and hence even without a hop constraint.

Observation 4.I. For any $h \geq 2$ and any $c \geq 1$, there is a two-player hybrid connectivity game with $\alpha=0$ and hop constraint $h$ whose price of anarchy is at least $c$.

Proof. Consider the example depicted in Figure 1(a). In the socially optimal state, player 1 connects to the access point, and player 2 connects to player 1 . In the worst Nash equilibrium, player 2 connects to the access point, and player 1 connects to player 2. In this case, the price of anarchy is $(M+1) / 3$. Since $M$ can be chosen arbitrarily, the observation follows for any fixed $c$.

Next, we consider hybrid connectivity games with $\alpha=1$. In the case of games without hop constraints, the price of anarchy is trivially 1 , because each player 


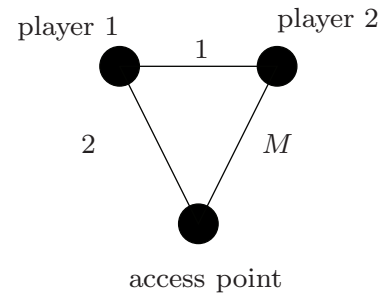

(a) The price of anarchy is unbounded in hybrid connectivity games with $\alpha=0$.

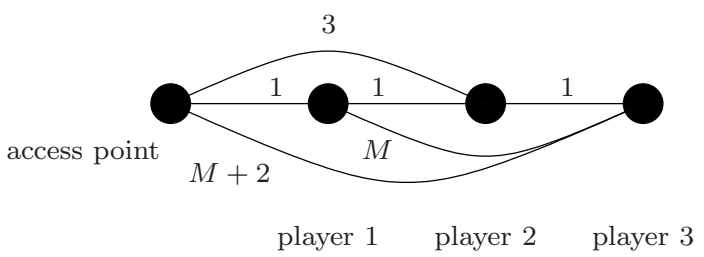

(b) The price of anarchy in hybrid connectivity games with $\alpha=1$ and hop constraint is unbounded.

Figure I. Worst-case instances.

is connected to the access point via a globally shortest path. Unfortunately, this positive result does not hold in games with hop constraints.

Observation 4.2. For any $h \geq 2$ and any $c \geq 1$, there is a three-player hybrid connectivity game with $\alpha=1$ and hop constraint $h$ whose price of anarchy is at least $c$.

Proof. Consider the example depicted in Figure 1(b), and assume that the hop constraint is 2 . Then, in the only equilibrium, player 1 connects to the access point, and players 2 and 3 to player 1 . In contrast to this, in the socially optimal state, player 2 connects to the access point, and player 3 to player 2 . In this case, the price of anarchy is $(M+4) / 8$. Since $M$ can be chosen arbitrarily, the observation follows for any fixed $c$.

In the aforementioned examples we did not make any assumptions about the cost function. However, in real-world wireless networks, devices are embedded into the Euclidean space $\mathbb{R}^{2}$ or $\mathbb{R}^{3}$, and costs are often assumed to depend on the Euclidean distance and the path-loss or distance power gradient $\beta \geq 1$. To be precise, the cost for a successful transmission of a data packet between two players $p_{1}$ and $p_{2}$ is $w\left(p_{1}, p_{2}\right)^{\beta}$, where $w\left(p_{1}, p_{2}\right)$ denotes the Euclidean distance between the two players. The distance power gradient usually ranges from 2 to 6 .

Next, we consider the price of anarchy in hybrid connectivity games with $\alpha=1$ that are embedded into Euclidean space. Given the distance power gradient $\beta$, we call these games $\beta$-embedded. We show that for such $\beta$-embedded games, the PoA is bounded above by $h^{\beta-1}$. Since the distance power gradient $\beta$ and the hop constraint are usually expected to be small constants, we conclude that the impact of selfish players is not too great and in particular does not depend on the number of players. 
Theorem 4.3. The price of anarchy in $\beta$-embedded hybrid connectivity games with $\alpha=1$ and hop constraint $h$ is bounded above by $h^{\beta-1}$.

Proof. In a Nash equilibrium $s$ of a $\beta$-embedded hybrid connectivity game with $\alpha=1$ and hop constraints, the cost of player $i$ is trivially bounded above by $w(i, a)^{\beta}$. Additionally, in a socially optimal state $s^{*}$, the cost of $i$ is bounded below by $h \cdot(w(i, a) / h)^{\beta}$. Thus, in every Nash equilibrium each player pays at most

$$
\frac{w(i, a)^{\beta}}{h \cdot(w(i, a) / h)^{\beta}}=h^{\beta-1}
$$

times what would be paid in a socially optimal state. Hence, the price of anarchy is bounded above by $h^{\beta-1}$.

\section{Conclusion}

By taking into account different kinds of payments, our analysis is a first step toward studying the impacts of selfishness stretching across the network and the data link layer. The major simplifying assumption in our model is that the energy consumption for sending packets between any pair of players is public knowledge and that there is a common valuation per unit of energy. Because of this assumption, reimbursements can be chosen in such a way that they precisely cover the cost of forwarding a packet.

One way to get rid of the assumption of global knowledge about the cost matrix might be simply to set a fixed price for forwarding packets, regardless of the required energy. In this case, only those nodes participate as relay stations that can forward packets along edges whose energy cost is not larger than the fixed price per packet. An alternative approach could be to use mechanisms like VCG to let the players truthfully report their cost values as described, for example, in [Anderegg and Eidenbenz 03, Eidenbenz et al. 07]. Both approaches have the problem that players might have an incentive to increase the number of packets they have to forward, because the payment that they receive is larger than the cost for forwarding a packet. For example, they might decrease the number of hops on their path to the base station in order to become more attractive to serve as a gateway for other nodes. That is, overpayments change the strategic behavior of the players and hence are not covered by our analysis.

We think that it would be an important step toward more practical models and a challenging open problem to investigate the effects of different payment schemes in a game-theoretic study. 


\begin{tabular}{|c|c|c|c|c|c|c|}
\hline & $a$ & $p_{1}$ & $p_{2}$ & $p_{3}$ & $p_{4}$ & $p_{5}$ \\
\hline$a$ & - & 20 & 58 & 60 & 97 & 85 \\
$p_{1}$ & 20 & - & 65 & 46 & 33 & 82 \\
$p_{2}$ & 58 & 65 & - & 48 & 71 & 15 \\
$p_{3}$ & 60 & 46 & 48 & - & 34 & 72 \\
$p_{4}$ & 97 & 33 & 71 & 34 & - & 18 \\
$p_{5}$ & 85 & 82 & 15 & 72 & 18 & - \\
\hline
\end{tabular}

Table I. A counterexample with $h=3$.

\section{Appendix: Proof of Theorem 2.3}

First, we present a hybrid connectivity game with $\alpha=0$ and hop constraint $h=3$ that does not possess a Nash equilibrium. In this game there are five players and a single access point. Edge weights are defined according to Table 1.

In order to prove that this game does not possess a Nash equilibrium, one could check every state of the game. Observe, however, that it suffices to consider a preference list for every player $p$ in which a player $p_{i}$ occurs before $p_{j}$ if $w\left(p, p_{i}\right)<$ $w\left(p, p_{j}\right)$. In case of ties, players can be listed in arbitrary order. Then each player wishes to be connected to the first player in its preference list under the assumption that this player is connected to the access point and that its own hop constraint is not violated. Additionally, in an equilibrium state no player is connected to some gateway that occurs after the access point $a$ in its preference list. Further, $p_{1}$ is always connected to the access point and therefore $p_{1}$ is always an appropriate gateway. Thus, we obtain the following preference lists:

$$
\begin{array}{ll}
p_{1}: & a \\
p_{2}: & p_{5}, p_{3}, a \\
p_{3}: & p_{4}, p_{1} \\
p_{4}: & p_{5}, p_{1} \\
p_{5}: & p_{2}, p_{4}, p_{3}, p_{1}
\end{array}
$$

In the following we do an exhaustive search over all possible states with respect to the shortened preference lists. Each line begins with the list of gateways chosen by the players $p_{2}, p_{3}, p_{4}, p_{5}$, followed by one out of several reasons why this state is not an equilibrium:

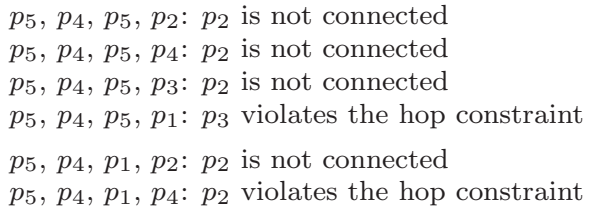


$p_{5}, p_{4}, p_{1}, p_{3}: p_{2}$ violates the hop constraint $p_{5}, p_{4}, p_{1}, p_{1}: p_{4}$ can decrease its cost

$p_{5}, p_{1}, p_{5}, p_{2}: p_{2}$ is not connected $p_{5}, p_{1}, p_{5}, p_{4}: p_{2}$ is not connected $p_{5}, p_{1}, p_{5}, p_{3}: p_{2}$ violates the hop constraint $p_{5}, p_{1}, p_{5}, p_{1}: p_{5}$ can decrease its cost $p_{5}, p_{1}, p_{1}, p_{2}: p_{2}$ is not connected $p_{5}, p_{1}, p_{1}, p_{4}: p_{2}$ violates the hop constraint $p_{5}, p_{1}, p_{1}, p_{3}: p_{2}$ violates the hop constraint $p_{5}, p_{1}, p_{1}, p_{1}: p_{3}$ can decrease its cost $p_{3}, p_{4}, p_{5}, p_{2}: p_{2}$ is not connected $p_{3}, p_{4}, p_{5}, p_{4}: p_{2}$ is not connected $p_{3}, p_{4}, p_{5}, p_{3}: p_{2}$ is not connected $p_{3}, p_{4}, p_{5}, p_{1}: p_{2}$ violates the hop constraint $p_{3}, p_{4}, p_{1}, p_{2}: p_{2}$ violates the hop constraint $p_{3}, p_{4}, p_{1}, p_{4}: p_{2}$ violates the hop constraint $p_{3}, p_{4}, p_{1}, p_{3}: p_{2}$ violates the hop constraint $p_{3}, p_{4}, p_{1}, p_{1}: p_{2}$ violates the hop constraint $p_{3}, p_{1}, p_{5}, p_{2}: p_{4}$ violates the hop constraint $p_{3}, p_{1}, p_{5}, p_{4}: p_{4}$ is not connected $p_{3}, p_{1}, p_{5}, p_{3}: p_{2}$ violates the hop constraint $p_{3}, p_{1}, p_{5}, p_{1}: p_{2}$ can decrease its cost

$p_{3}, p_{1}, p_{1}, p_{2}: p_{5}$ violates the hop constraint $p_{3}, p_{1}, p_{1}, p_{4}: p_{3}$ can decrease its cost $p_{3}, p_{1}, p_{1}, p_{3}: p_{3}$ can decrease its cost $p_{3}, p_{1}, p_{1}, p_{1}: p_{3}$ can decrease its cost

$a, p_{4}, p_{5}, p_{2}: p_{3}$ violates the hop constraint

$a, p_{4}, p_{5}, p_{4}: p_{3}$ is not connected

$a, p_{4}, p_{5}, p_{3}: p_{3}$ is not connected

$a, p_{4}, p_{5}, p_{1}: p_{3}$ violates the hop constraint

$a, p_{4}, p_{1}, p_{2}: p_{4}$ can decrease its cost

$a, p_{4}, p_{1}, p_{4}: p_{5}$ can decrease its cost

$a, p_{4}, p_{1}, p_{3}: p_{5}$ violates the hop constraint

$a, p_{4}, p_{1}, p_{1}: p_{4}$ can decrease its cost

$a, p_{1}, p_{5}, p_{2}: p_{2}$ can decrease its cost

$a, p_{1}, p_{5}, p_{4}: p_{4}$ is not connected

$a, p_{1}, p_{5}, p_{3}: p_{4}$ violates the hop constraint

$a, p_{1}, p_{5}, p_{1}: p_{2}$ can decrease its cost

$a, p_{1}, p_{1}, p_{2}: p_{2}$ can decrease its cost

$a, p_{1}, p_{1}, p_{4}: p_{2}$ can decrease its cost

$a, p_{1}, p_{1}, p_{3}: p_{2}$ can decrease its cost

$a, p_{1}, p_{1}, p_{1}: p_{2}$ can decrease its cost

In order to extend the example to arbitrary hop constraints $h>3$, one simply replaces the former access point by a sequence of $h-3$ players and attaches a new access point to this sequence. The weights are chosen in such a way that the new players always line up and such that none of the old players connect to a new one.

This completes the proof. 
Acknowledgments. This work was supported in part by the Ultra High-Speed Mobile Information and Communication Research Cluster (UMIC) established under the excellence initiative of the German government, and by the DFG under grant Kr 2332/1-2 within the Emmy Noether program. A preliminary version of this paper appeared in Proceedings of the Third International Workshop on Internet and Network Economics (WINE) [Ackermann et al. 07].

\section{References}

[Ackermann et al. 07] H. Ackermann, P. Briest, A. Fanghänel, and B. Vöcking. "Who Should Pay for Forwarding Packets?" In Internet and Network Economics: Third International Workshop, WINE 2007, San Diego, CA, USA, December 12-14, 2007, Proceedings, Lecture Notes in Computer Science 4858, pp. 208-219. New York: Springer, 2007.

[Albers et al. 06] S. Albers, S. Eilts, E. Even-Dar, Y. Mansour, and L. Roditty. "On Nash Equilibria for a Network Creation Game." In Proceedings of the 17th Annual ACM-SIAM Symposium on Discrete Algorithms, pp. 89-98. New York: ACM Press, 2006.

[Altman and Altman 03] E. Altman and Z. Altman. "S-Modular Games and Power Control in Wireless Networks." IEEE Transactions on Automatic Control 48:5 (2003), 839-842.

[Anderegg and Eidenbenz 03] L. Anderegg and S. Eidenbenz. "Ad Hoc-VCG: A Truthful and Cost-Efficient Routing Protocol for Mobile Ad Hoc Networks with Selfish Agents." In Proceedings of the 9th Annual International Conference on Mobile Computing and Networking, pp. 245-259. New York: ACM Press, 2003.

[Anshelevich et al. 03] E. Anshelevich, A. Dasgupta, E. Tardos, and T. Wexler. "NearOptimal Network Design with Selfish Agents." In Proceedings of the 35th Annual ACM Symposium on Theory of Computing, pp. 511-520. New York: ACM Press, 2003.

[Bala and Goyal 00] V. Bala and S. Goyal. "A Non-cooperative Theory of Network Formation." Econometrica 68:5 (2000), 1181-1229.

[Corbo and Parkes 05] J. Corbo and D. Parkes. "The Price of Selfish Behavior in Bilateral Network Formation." In Proceedings of the Twenty-Fourth Annual ACM Symposium on Principles of Distributed Computing, pp. 99-107. New York: ACM Press, 2005.

[Demaine et al. 07] E. D. Demaine, M. Hajiaghayi, H. Mahini, and M. Zadimoghaddam. "The Price of Anarchy in Network Creation Games." In Proceedings of the Twenty-Sixth Annual ACM Symposium on Principles of Distributed Computing, pp. 292-298. New York: ACM Press, 2007.

[Eidenbenz et al. 07] S. Eidenbenz, L. Anderegg, and R. Wattenhofer. "IncentiveCompatible, Energy-Optimal, and Efficient Ad Hoc Networking in a Selfish Milieu." 
In Proceedings of the 40th Annual Hawaii International Conference on System Sciences, pp. 293-302. Washington, DC: IEEE Computer Society, 2007.

[Eidenbenz et al. 03] S. Eidenbenz, V. S. A. Kumar, and S. Zust. "Equilibria in Topology Control Games for Ad Hoc Networks." In Proceedings of the 2003 Joint Workshop on Foundations of Mobile Computing, pp. 2-11. New York: ACM Press, 2003.

[Fabrikant et al. 03] A. Fabrikant, A. Luthra, E. Maneva, C. H. Papadimitriou, and S. Shenker. "On a Network Creation Game." In Proceedings of the Twenty-Second Annual Symposium on Principles of Distributed Computing, pp. 347-351. New York: ACM Press, 2003.

[Frodigh et al. 01] M. Frodigh, S. Parkvall, C. Roobol, P. Johansson, and P. Larsson. "Future-Generation Wireless Networks." IEEE Personal Communications 8:5 (2001), 10-17.

[Heikkinen 06] T. Heikkinen. "A Potential Game Approach to Distributed Power Control and Scheduling." Computer Networks 50:13 (2006), 2295-2311.

[Jakobsson et al. 03] M. Jakobsson, J. P. Hubaux, and L. Buttyán. "A Micro-payment Scheme Encouraging Collaboration in Multi-hop Cellular Networks." In Financial Cryptography: 7th International Conference, FC 2003, Guadeloupe, French West Indies, January 27-30, 2003, Revised Papers, Lecture Notes in Computer Science 2742, pp. 15-33. Berlin: Springer, 2003.

[Krishnaswamy 02] D. Krishnaswamy. "Game Theoretic Formulations for NetworkAssisted Resource Management in Wireless Networks." In Proceedings of the 56th IEEE Vehicular Technology Conference VTC Fall 2002, Vol. 3, pp. 1312-1316. Los Alamitos, CA: IEEE Press, 2002.

[Singh et al. 98] S. Singh, M. Woo, and C. S. Raghavendra. "Power-Aware Routing in Mobile Ad Hoc Networks." In Proceedings of the 4th Annual ACM/IEEE International Conference on Mobile Computing and Networking, pp. 181-190. New York: ACM Press, 1998.

[Srivastava et al. 05] V. Srivastava, J. Neel, A. B. Mackenzie, R. Menon, L. A. Dasilva, J. E. Hicks, J. H. Reed, and R. P. Gilles. "Using Game Theory to Analyze Wireless Ad Hoc Networks." IEEE Communications Surveys and Tutorials 7:4 (2005), 46-56.

[Tang et al. 05] J. Tang, G. Xue, and W. Zhang. "Interference-Aware Topology Control and QoS Routing in Multi-channel Wireless Mesh Networks." In Proceedings of the 6th ACM International Symposium on Mobile Ad Hoc Networking and Computing, pp. 68-77. New York: ACM Press, 2005.

[Zhong et al. 05] S. Zhong, E. L. Li, Y. G. Liu, and Y. Yang. "On Designing IncentiveCompatible Routing and Forwarding Protocols in Wireless Ad-Hoc Networks: An Integrated Approach Using Game Theoretical and Cryptographic Techniques." In Proceedings of the 11th Annual International Conference on Mobile Computing and Networking, pp. 117-131. New York: ACM Press, 2005. 
Heiner Ackermann, Fraunhofer Institute for Industrial Mathematics, Fraunhofer-Platz 1, 67663 Kaiserslautern, Germany (heiner.ackermann@itwm.fraunhofer.de)

Patrick Briest, Department of Computer Science, University of Paderborn, Fürstenallee 11, 33102 Paderborn, Germany (patrick.briest@upb.de)

Alexander Fanghänel, Department of Computer Science, RWTH Aachen, Ahornstr. 55, 52074 Aachen, Germany (fanghaenel@cs.rwth-aachen.de)

Berthold Vöcking, Department of Computer Science, RWTH Aachen, Ahornstr. 55, 52074 Aachen, Germany (voecking@cs.rwth-aachen.de)

Received March 31, 2008; accepted June 25, 2009. 